\title{
A Clinical Trial of Therapeutic Electrical Stimulation for Amyotrophic Lateral Sclerosis
}

\author{
Ikuko Handa, Noboru Matsushita, Kouji Ihashi, Ryo \\ Yagi, Ruriko Mochizuki*, Hiroshi Mochizuki*, Yumiko \\ Abe*, Yusei Shiga*, Nozomu Hoshimiya $\dagger$, Yasuto \\ ITOYAMA* and YASUNOBU HANDA \\ Department of Restorative Neuromuscular Surgery and \\ Rehabilitation, Graduate School of Medicine, Tohoku \\ University, *Department of Neurology, Tohoku University \\ School of Medicine, †Division of Engineering, Tohoku \\ University Graduate School, Sendai 980-77
}

Handa, I., Matsushita, N., Ihashi, K., Yagi, R., Mochizuki, R., Mochizuki, H., Aвe, Y., Shiga, Y., Hoshimiya, N., Itoyama, Y. and Handa, Y. A Clinical Trial of Therapeutic Electrical Stimulation for Amyotrophic Lateral Sclerosis. Tohoku J. Exp. Med., 1995, 175 (2), 123-134 — This paper describes the effects of therapeutic electrical stimulation (TES) on the wasting muscles in a patient with amyotrophic lateral sclerosis. The patient is a 47 -year-old male, and he has a history of progressive muscle weakness and atrophy, affected more in the right side. Percutaneously indwelling intramuscular electrodes were implanted to the affected muscles in the right upper and lower extremities but no electrode in the corresponding left region. Within a month of TES therapy, a rapid improvement of extremity motion appeared in the TES treated side. Long-term application of TES more than 3 months increased the strength of the muscle which had been evidently weaker than the non-treated side. CT findings of both the upper and lower extremities with TES therapy showed an increase in the density and a reduction in the moth-eaten image. An increase in the thickness of the muscles was also observed in the TES treated side while deterioration was observed in the muscles on the non-treated side.__ amyotrophic lateral sclerosis; therapeutic electrical stimulation; intramuscular electrode; extremity

Amyotrophic lateral sclerosis (ALS) is, in general, a rapidly progressive untreatable disease affecting both the upper (the lateral column) and the lower (the anterior horn cells) motor neurons. Recently, therapeutic trials for treating motor neuron diseases including ALS have been reported for humans as well as animals (Bensimon et al. 1994; Matsumoto et al. 1994a, b). However, we have not found an article describing the therapeutic effect of electrical stimulation on ALS affected muscles of the patient.

Electrical stimulation to the paretic muscles involved in upper motor neuron disorders, such as cerebrovascular accident and spinal cord injury, induces some

Received January 10, 1995; accepted for publication January 11, 1995. 
therapeutic effects, i.e., an improvement of atrophy and an increase in muscle strength, a reduction of spasticity and so on. We experienced a marked improvement in the function of the paretic muscles caused by brachial plexus injury (Kameyama et al., in press), a lower motor neuron disorder. It is likely that electrical stimulation has therapeutic effects on the wasting muscles in both upper and lower motor neuron disorders.

This paper describes therapeutic electrical stimulation (TES) on the affected muscles of an ALS patient.

\section{Subject And Methods}

\section{Subject}

A 47-year-old male patient with a 5-year history of progressive muscle waste by ALS.

Present illness. The patient recognized difficult dorsiflexion of the right big toe during gait in June, 1990. Toward the end of that year, atrophy of the hand and muscle weakness of the knee extensor and ankle dorsiflexor on the right appeared. The muscle weakness slowly progressed and affected the contralateral (left) lower extremity until February, 1992 when the patient could only walk using foot orthoses and a cane. Muscle waste of the right upper extremity gradually progressed and writing became impossible by October, 1993. In 1994, he had muscle weakness in the left upper extremity and severe difficulty in walking. Due to progressive muscle weakness, walking became impossible and he started to use a wheel chair from February, 1994. Thereafter, dyspnea, dysphagia and weakness of facial muscles appeared.

Family history. His parents and both his brother and sister are healthy. No hereditary disease is found in his family.

Physical examination. There was no particular abnormality in general medical condition except tachycardia and tachypnea. Neurological examination revealed muscle atrophy of the face, trunk and extremities and fasciculation of the muscles was observed in the tongue, the four extremities and the upper chest wall. Atrophy and weakness of the muscle were dominant in the distal part of the extremity. Such muscle waste was much severer on the right side and the lower extremities. No sensory disturbance was found.

Since respiratory movement by the intercostal muscles and diaphragm was also affected, vital capacity and blood gas findings before respirator application exhibited changes in hypoventilation.

Neurogenic changes, such as fasciculation voltage, were observed by electromyographic analysis.

Creatinin phosphokinase showed the highest value of 1482 IU in December, 1990. Before TES application in July, 1994, the value was 229 IU. 


\section{Electrodes and TES system}

The percutaneously indwelling intramuscular electrode (Nippon Seisen Co. Ltd., Osaka) was used to apply stimulus current to the muscle branches of the peripheral nerve. Details of configuration of this electrode are described elsewhere (Handa et al. 1989). We utilized the FES system (NEC San-ei Co. Ltd., Tokyo), which we developed (Ohkubo et al. 1989; Handa et al. 1992), as a TES system. This FES system contains an automatic training mode where the duty ratio and duration of cyclic stimulation can be adjusted. Stimulation parameters such as pulse width, amplitude and stimulation frequency are also described elsewhere (Handa and Hoshimiya 1987).

\section{Electrode implantation and stimulation pattern}

Electrode implantation was achieved under general anesthesia. Prior to the implantation, tracheotomy was performed to improve respiratory function by a respirator.

In order to examine the effect of TES on the affected muscles, percutaneous intramuscular electrodes were implanted in the muscles and muscle branches of the peripheral nerves of the right upper and lower extremities, but no electrodes were implanted in the left side. The muscles and nerves to which the electrodes were implanted are listed in Table 1.

Stimulation patterns of TES for the upper and lower extremities consisted of two phases, i.e., an extension phase and a flexion phase.

During the extension phase of the upper extremity, the shoulder joint was abducted through the stimulation of the deltoid, supraspinatus, infraspinatus and serratus anterior muscles. The elbow was extended by the triceps brachii stimulation and the hand was opened by the stimulation of the extensor digitorum (Fig. 1a).

TABLE 1. Muscles and nerves stimulated

Right upper extremity
1: supraspinatus
7: brachialis
2 : infraspinatus
8 : brachioradialis
3 : deltoid
9 : extensor carpi radialis brevis
4 : serratus anterior
10 : extensor digitorum
5 : triceps brachii
11 : flexor digitorum profundus
6 : biceps brachii
12 : abductor pollicis brevis

Right lower extremity
1 : inferior gluteal nerve
4 : biceps femoris
2: superior gluteal nerve
5: tibial nerve
3 : femoral nerve 
For the flexion phase, the biceps brachii, brachialis and brachioradialis were activated and simultaneously cylindrical grasp movement of the hand was achieved by stimulation of the flexor digitorum profundus, abductor pollicis brevis and extensor carpi radialis brevis.

The extension phase of the lower extremity was achieved by stimulating the femoral, inferior gluteal and tibial nerves, which innervated to the quadriceps femoris, gluteus maximus and triceps surae muscles, respectively. The lateral hamstring muscles were stimulated during the flexion phase.

TES schedule for this patient was:

$\begin{array}{lr}\text { 1st week } & 5 \mathrm{~min} \times 3 \text { times/day } \\ \text { 2nd-3nd week } & 7 \mathrm{~min} \times 6 \text { times/day } \\ \text { 4th-6th week } & 10 \mathrm{~min} \times 6 \text { times/day } \\ \text { 7th week-present } & 10 \mathrm{~min} \times 7 \text { times/day }\end{array}$

\section{Assessment of TES effects}

Changes in muscle force were evaluated by Daniel's manual muscle testing (Daniels and Worthingham 1986). Joint movements of the upper and lower extremities were analyzed using a three dimensional motion analyzer (APAS Ariel, Trabuco Canyon, CA, USA). Computed tomography (CT) was used to detect the changes in volume and quality of the muscle.

\section{RESUlts}

During the month from the start of TES therapy, improvements in the motion of the right upper and lower extremities were observed.

Within two weeks of TES therapy, the patient could easily perform a pitching motion and a reaching motion to the occipital region of his head and to the contralateral auricle (Fig. 1b). These movements were very hard to perform 3 months before the TES application. Thereafter, the patient could clap his hands powerfully and tie strings with both hands (Fig. 1c). This suggests that motion improvement started at the proximal part of the upper extremity (shoulder and elbow) and reached the distal part (hand and wrist). During 4 months of TES applications, further and gradual improvement in the right upper extremity function was observed and the laterality of the upper extremity function became smaller although the motion and force were still better and stronger on the left side.

Fig. 2 shows the motion analysis of voluntary elbow flexion, where the patient was requested to flex the elbow with a constant speed. A stick picture of the elbow flexion showed apparent instability of the movement before TES treatment, with more stability after 3 months of TES therapy. The fluctuation of angular velocity was much smaller in the movement of the elbow after TES therapy than before.

Hip elevation (bridging motion) was performed shortly after two weeks of the 

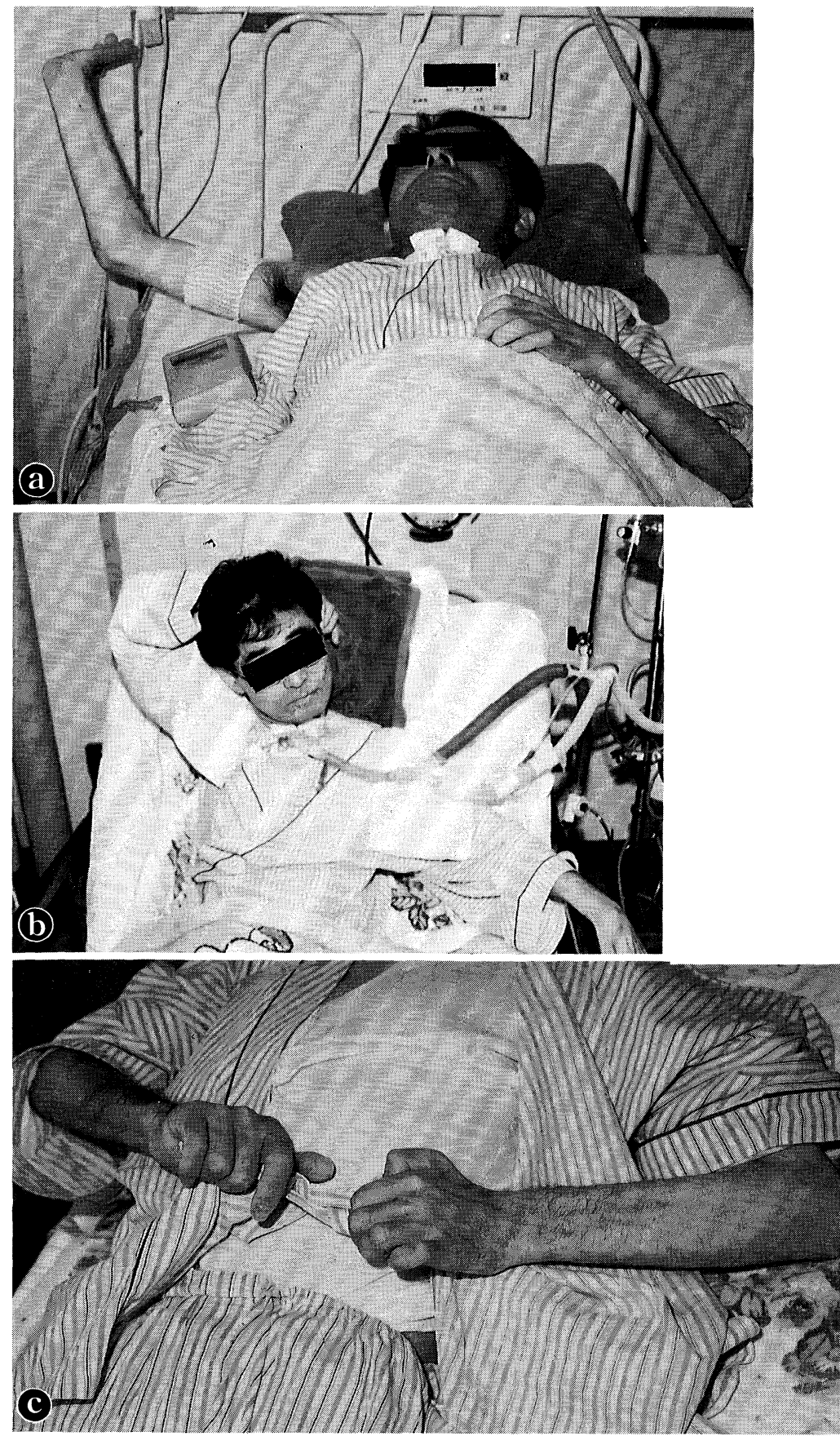

Fig. 1. Movement induced by TES and effects of TES for the upper extremity. a : Shoulder abduction with elbow and hand extension controlled by TES. $\mathrm{b}$ : Active reaching motion after 2 weeks of TES therapy.

c: The patient could tie strings after 6 weeks of TES therapy. 
I. Handa et al.

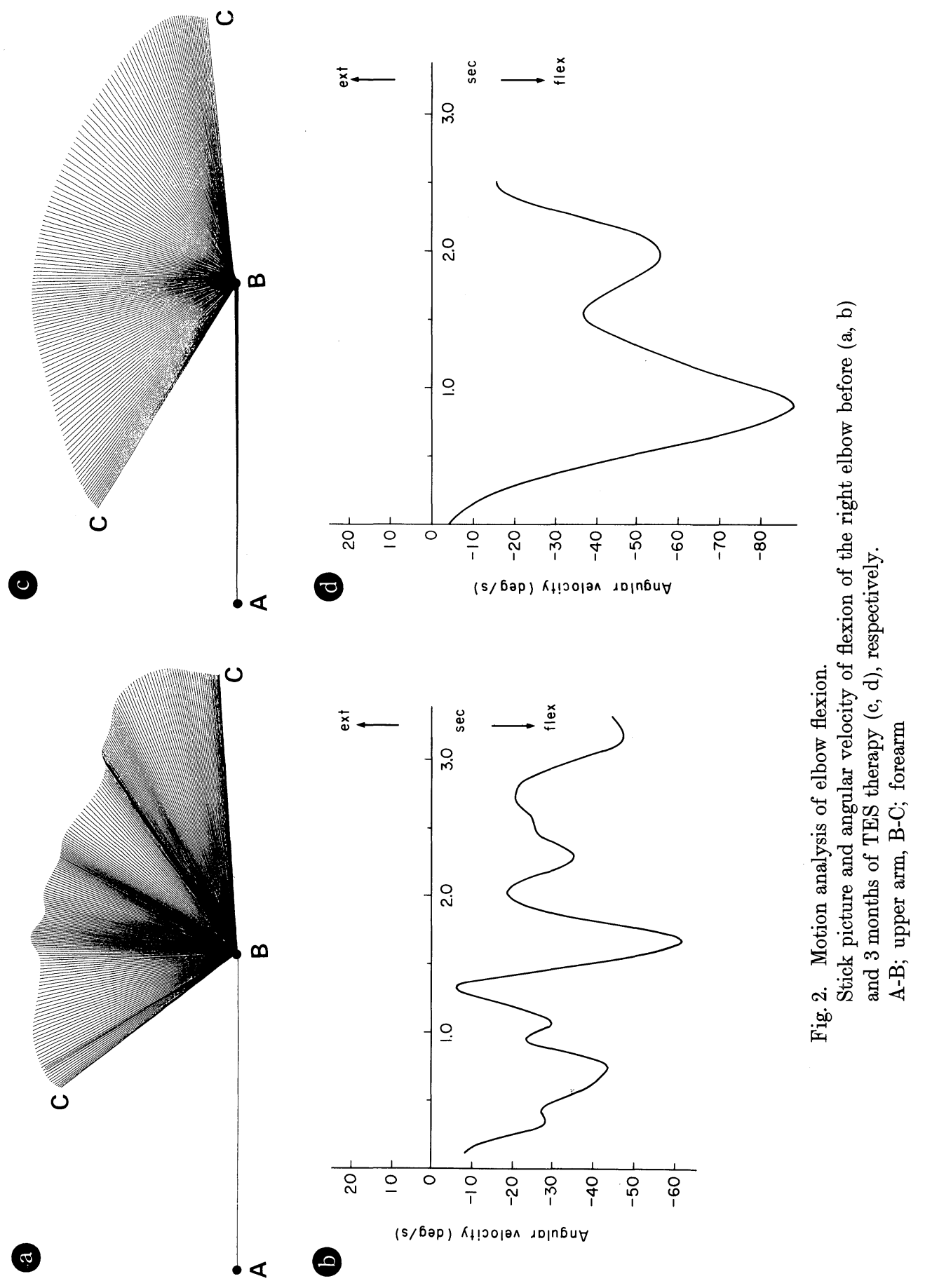


onset of TES therapy. Since the height and time of hip elevation became higher and longer, it became easier for his helper to assist with bed slippers and to change shorts or pajamas (Fig. 3). The total force of leg extension induced by simultaneous extension of the hip and knee joints was stronger on the left before TES application. Through 3 months of TES therapy, the extension force showed an increase on the right and a decrease on the left. Finally, laterality of the muscle power was almost reversed. In addition, a gradual increase in the extension force of the right leg resulted in a decrease in the force needed to assist the patient in a standing transfer between a bed and a wheel chair.

Comparison of MMT before and during TES therapy is shown in Table 2. Before TES therapy, the MMT grades were dominant in both the upper and lower extremities in the left side. Although the MMT grade for each muscle was almost unchanged even after more than 3 months of TES treatment, the difference in muscle force between both extremities became smaller. This is likely because the force of the right extremity muscles increased within the same MMT grade range.

In the lower extremities, however, the knee extension power on the right became larger than that on the left. Although the MMT grade for both knee extensions before TES application was 2, where the patient could extend the knee under a gravity free condition, the actual force of extension was apparently stronger in the left leg. At the 3rd month of the TES therapy, MMT grades were 2 and 2 - on the right and left side, respectively. Full range extension of the knee joint became impossible under a gravity free condition on the left.

Fig. 4 shows CT findings for the bilateral quadriceps femoris and gluteus maximus. Before TES treatment, both quadriceps femoris show typical findings of muscle degeneration (Fig. 4a). A moth-eaten image was observed within the atrophied muscles. This indicates an increase in adipose connective tissue.

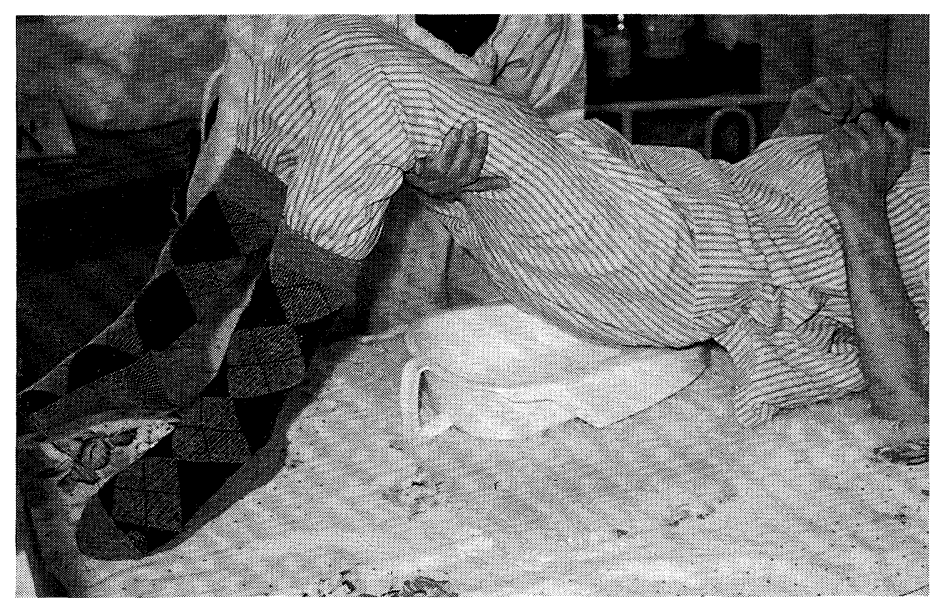

Fig. 3. Effect of TES for the lower extremity.

Active bridging motion after 3 months of TES therapy. 
TABLE 2. MMT grades before and after TES therapy

\begin{tabular}{|c|c|c|c|c|c|c|}
\hline \multirow{2}{*}{ Muscles } & \multicolumn{2}{|c|}{ Before } & \multicolumn{2}{|c|}{1 month } & \multicolumn{2}{|c|}{3.5 months } \\
\hline & Right & Left & Right & Left & Right & Left \\
\hline \multicolumn{7}{|c|}{ Upper extremities } \\
\hline \multicolumn{7}{|l|}{ Shoulder } \\
\hline Flexors & 4 & 4 & 4 & 4 & 4 & 4 \\
\hline Abductors & 4 & 4 & 4 & 4 & 4 & 4 \\
\hline \multicolumn{7}{|l|}{ Elbow } \\
\hline Flexors & 4 & 4 & 4 & 4 & 4 & 4 \\
\hline Extensors & 4 & 4 & 4 & 4 & 4 & 4 \\
\hline \multicolumn{7}{|l|}{ Wrist } \\
\hline Extensors & 4 & 4 & 4 & 4 & 4 & 4 \\
\hline Flexors & 3 & 4 & 3 & 4 & 3 & 3 \\
\hline \multicolumn{7}{|l|}{ Finger } \\
\hline Extensors & 4 & 4 & 4 & 4 & 4 & 4 \\
\hline Flexors & 3 & 3 & 4 & 4 & 4 & 4 \\
\hline \multicolumn{7}{|c|}{ Lower extremities } \\
\hline \multicolumn{7}{|l|}{ Hip } \\
\hline Extensors & 2 & 2 & & & 2 & $2-$ \\
\hline Flexors & $1+$ & $2-$ & $1+$ & 2 & 1 & $\overline{1}$ \\
\hline \multicolumn{7}{|l|}{ Knee } \\
\hline Extensors & 2 & 2 & 2 & 2 & 2 & $2-$ \\
\hline Flexors & $1+$ & $2-$ & 1 & $2-$ & 1 & 1 \\
\hline \multicolumn{7}{|l|}{ Ankle } \\
\hline $\begin{array}{l}\text { Planter- } \\
\text { flexors }\end{array}$ & $2-$ & $2-$ & & & $2-$ & $1+$ \\
\hline
\end{tabular}

Decrease in density of the muscle itself was also observed.

TES therapy for 3 months caused an apparent change in the CT image as shown in Fig. 4b. Although the density of the muscle increased in both quadriceps femoris muscles, it was rather dominant in the TES treated muscle. The moth-eaten image observed in the stimulated muscles markedly decreased. The shape of the cross section of the right thigh became round from oval through long-term TES and its circumference decreased. The contralateral thigh showed no change in its oval shape and only a slight increase in the circumference. Furthermore, muscle belly of the right gluteus maximus became thicker with increased density by TES therapy while the left gluteus maximus showed a reduction in its thickness and decreased density (Fig. 5a, b).

\section{Discussion}

TES application on an ALS patient for 4 months brought therapeutic effects to function and morphology of the affected muscle. In the process of functional improvement by TES, two phases may exist. One is an relatively acute phase and the other is a chronic one. The former was observed within only a month from the onset of TES therapy. The latter appeared afterwards. 

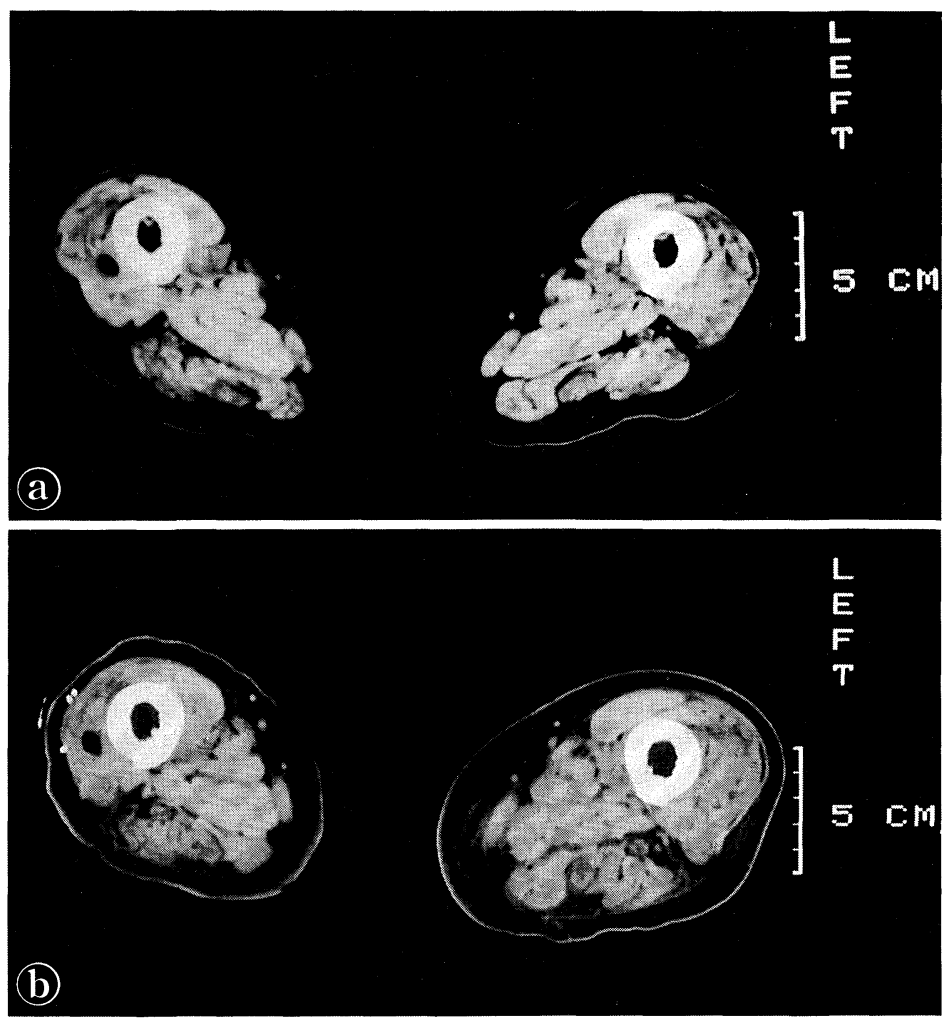

Fig. 4. CT image before and after 3 months of TES therapy.

Cross section of the bilateral thighs before (a) and after (b) TES therapy.

It was found that TES using intramuscular electrodes showed two kinds of therapeutic effect on the paralyzed extremities of upper motor neuron disorders, i.e., efferent and afferent effects (Handa and Sakurai 1993). Efferent effects which were provided by stimulation of alpha-motoneurons were increases in volume and force on the muscles stimulated (Peckham et al. 1976; Bajd et al. 1989). Afferent effects were obtained by the stimulation of afferent nerves from the muscle, fascia and/or tendon. Since the threshold of gIa fibers for electrical stimulation is lower than that of alpha-motoneurons, a stimulus current which evokes muscle contraction can always elicit afferent volleys of gIa fibers. This results in a reduction of spasticity through reciprocal inhibition of the antagonist of the activated muscle by electrical stimulation. Such a reduction of spasticity by cyclic electrical stimulation has also been reported by many investigators (Levine et al. 1952; Kralj and Bajd 1989). In general, the afferent effects appeared much faster than the efferent effects.

Neurologically, an ALS process can be characterized by signs of upper motor neuron deficit and/or lower motor neuron loss. The former are the pyramidal 

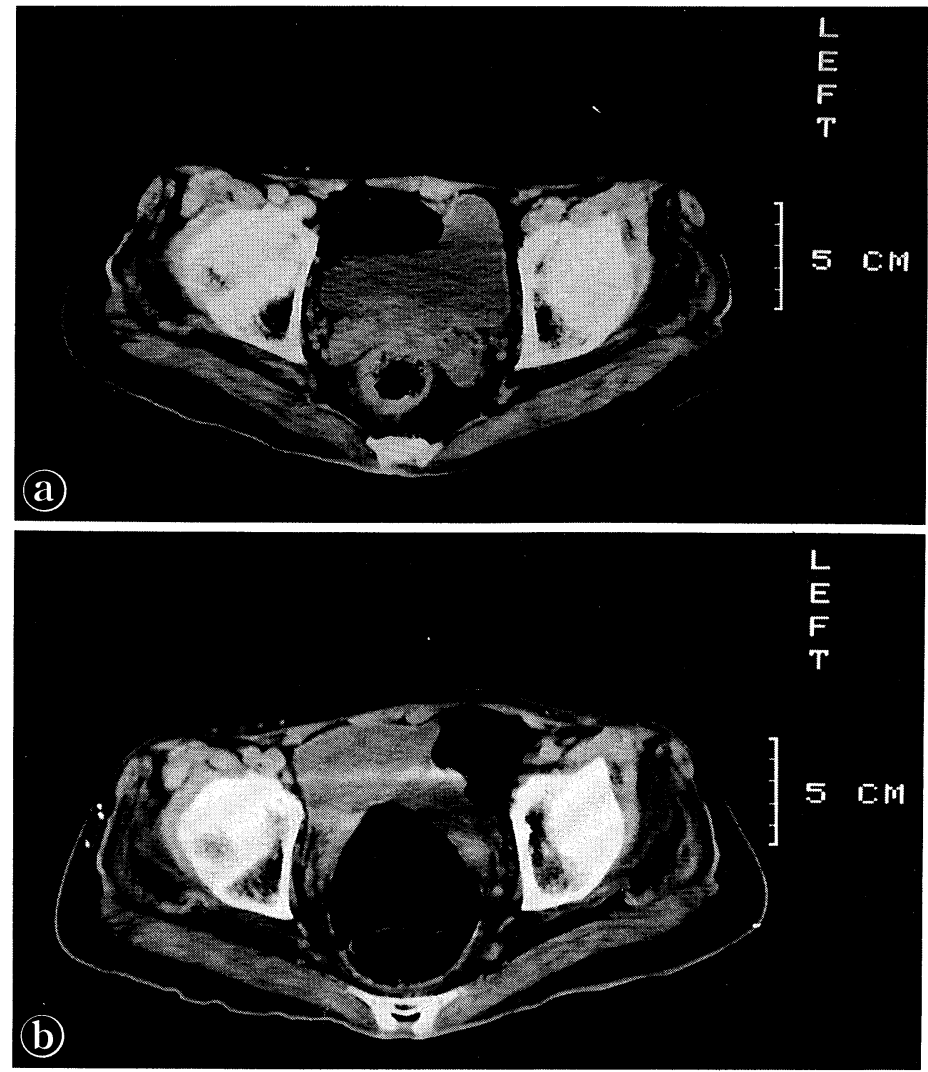

Fig. 5. CT image of the pelvis.

Before (a) and 3 months after (b) TES therapy.

tract signs, i.e., spasticity, pathological reflex and hyperreflexia. The latter are the denervation signs such as weakness, atrophy and fascicular contraction of the muscle. In this patient, both signs were observed when TES started. Since spasticity in ALS is not usually dominant as compared with muscle waste, it seems that spasticity constitutes only a small portion of motor disturbance (Sinaki 1988). However, joint movements induced by a certain muscle group may be prevented by even a slight increase in spasticity of its antagonists. A relatively rapid improvement in upper extremity motion within a month might be due to a reduction of spasticity, an afferent effect of TES.

However, one can argue that recovery from respiratory dysfunction by respirator application may cause such a rapid improvement in motor function of the extremities. In fact, blood gasses became much improved 2 weeks after the onset of artificial respiration. Further investigations will be necessary in order to clarify this.

It has been reported that electrical stimulation to the denervated muscle 
improves muscle atrophy (Harbison et al. 1971). Our TES therapy, however, is ineffective for denervated muscles because the stimulus parameters such as pulse amplitude and duration are not strong enough to evoke contraction of the denervated muscle. TES effects on the lower motor neuron involvement in the process of denervation are still unknown. This implies that we may expect the efferent effects of TES through the stimulation of the survived alpha-motor neurons. In fact, the long-term comparison of the movement and muscle strength between TES treated and non-treated extremities of this ALS patient showed a decrease in laterality in the upper extremity and a reversal of laterality in the lower extremities. It is likely that these relatively long-term effects are due to the efferent effects of TES.

The morphological changes shown in CT follow-up support the functional improvement of the extremities through a long-term TES application. It is known that the moth-eaten change with a decreased density was observed in a CT image of the muscles affected by ALS (Hawley et al. 1984). These changes are always progressive in this disease. Therefore, it is likely that a reduction of moth-eaten appearance and an increase in density is due to a long-term electrical stimulation. An increase in thickness of the muscle belly accompanied by an increased density which was observed in the TES treated gluteal muscle may be also responsible to the efferent effects of electrical stimulation.

In order to discuss the effects of TES on the wasted muscles of ALS more precisely, further follow-up study with more patients is required.

\section{Acknowledgments}

We wish to express our thanks to Dr. S. Ono and Prof. H. Fukuda of Institute of Development Aging and Cancer for their helpful discussion. Thanks are tendered to members of Sendai FES Project and graduate students of the Department of Restorative Neuromuscular Surgery and Rehabilitation for their help.

\section{References}

1) Bajd, T., Kralj, A., Turk, R., Benko, H. \& Šega, J. (1989) Use of functional electrical stimulation in the rehabilitation of patients with incomplete spinal cord injuries. $J$. Biomed. Eng., 11, 96-102.

2) Bensimon, G., Lacomblez, L., Meininger, V. \& ALS/Riluzole Study Group (1994) A controlled trial of riluzole in amyotrophic lateral sclerosis. N. Engl. J. Med., 330, 585-591.

3) Daniels, L. \& Worthingham, C. (1986) Muscle Testing. 5th ed., Saunders.

4) Handa, Y. \& Hoshimiya, N. (1987) Functional electrical stimulation for the control of the upper extremities. Med. Prog. Technol., 12, 51-63.

5) Handa, Y. \& Sakurai, M. (1993) Functional electrotherapy using percutaneously indwelling intramuscular electrodes. Yakuji Shinpo, No. 1754, 714-719. (in Japanese)

6) Handa, Y., Hoshimiya, N., Iguchi, Y. \& Oda, T. (1989) Development of percutaneous intramuscular electrode for multichannel FES system. IEEE Trans. Biomed. Eng., 36, 705-710. 
7) Handa, Y., Handa, T., Ichie, M., Murakami, H., Hoshimiya, N., Ishikawa, S. \& Ohkubo, K. (1992) Functional electrical stimulation (FES) systems for restoration of motor function of paralyzed muscle-versatile systems and a portable system. Front. Med. Biol. Engng., 4, 241-255.

8) Harbison, G.J., Teng, C., Reyes, T. \& Reyes, O. (1971) Effect of electrical stimulation on denervated muscle of rat. Arch. Phys. Med. Rehabil., 52, 516-522.

9) Hawley, R.J., Schellinger, D. \& O'Doherty, D.S. (1984) Computed tomographic patterns of muscles in neuromuscular diseases. Arch. Neurol., 41, 383-387.

10) Kameyama, J., Sakurai, M. \& Handa, Y. Therapeutic electrical stimulation using percutaneous multiple electrodes for the purpose of improvement to the upper limb palsy due to brachial plexus injury. Seikeigeka (in press) (in Japanese)

11) Kralj, A.R. \& Bajd, T. (1989) Influence of electrical stimulation on spasticity in spinal cord injured patients. In: Functional Electrical Stimulation: Standing and Walking after Spinal Cord Injury, edited by A.R. Kralj \& T. Bajd, CRC Press, Inc., Boca Raton-Florida, pp. 37-48.

12) Levine, M.G., Knott, M. \& Kabat, H. (1952) Relaxation of spasticity by electrical stimulation of antagonist muscles. Arch. Phys. Med., 33, 668-673.

13) Matsumoto, H., Ikeda, K., Holmlund, T., Green, T., Cedar baum, J.M., Wong, V. \& Lindsay, R.M. (1994a) The effects of ciliary neurotrophic factor on motor dysfunction in wobbler mouse motor neuron disease. Ann. Neurol., 36, 142-148.

14) Matsumoto, H., Ikeda, K., Klinkosz, B., Cedarbaum, J.M., Wong, V. \& Lindsay, R.M. (1994b) Arrest of motor neuron disease in wobbler mice cotreated with CNTF and BDNF. Science, 265, 1107-1110.

15) Ohkubo, K., Hoshimiya, N. \& Handa, Y. (1989) Development of second generation FES system for practical use. Proc. 11th Ann. Conf. IEEE/EMBS, 1002-1003.

16) Peckham, P.H., Mortimer, J.T. \& Marsolais, E.B. (1976) Alteration in the force and fatigability of skeletal muscle in quadriplegic humans following exercise induced by chronic electrical stimulation. Clin. Orthop., 114, 326-334.

17) Sinaki, M. (1988) Exercise and rehabilitation measure in amyotrophic lateral sclerosis. In: Amyotrophic Lateral Sclerosis, edited by T. Tsubaki \& Y. Yase, Elsevier Scientific Publishers B.V., Amsterdam-London-New York, pp. 343-368. 